\title{
Kernos
}

Revue internationale et pluridisciplinaire de religion grecque antique

8| 1995

Varia

\section{Revue des actes de colloques}

\section{(2) OpenEdition \\ Journals}

\section{Édition électronique}

URL : http://journals.openedition.org/kernos/629

DOI : $10.4000 /$ kernos.629

ISSN : 2034-7871

\section{Éditeur}

Centre international d'étude de la religion grecque antique

Édition imprimée

Date de publication : 1 janvier 1995

Pagination : 323-326

ISSN : 0776-3824

\section{Référence électronique}

«Revue des actes de colloques », Kernos [En ligne], 8| 1995, mis en ligne le 12 avril 2011, consulté le 24 septembre 2020. URL : http://journals.openedition.org/kernos/629 ; DOI : https://doi.org/10.4000/ kernos.629 


\title{
3. Actes de colloques
}

\author{
Jaime Alvar, Carmen Blanquez, Carlos G. Wagner (éds), Formas de \\ difusión de las religiones griegas. Segundo encuentro de ARYS. \\ Jarandilla de la Vera, Diciembre 1990, Madrid, 1993, VII+289 p.
}

Pilar FERNANDEZ URIEL, La evolución mitológica de un mito: la abeja, p. 133-159

Ana María VAZQUEz HOYS, Perduración y cambio de significado de la serpiente en las monedas antiguas, p. 161-171

Domingo PLACiDO, Cresmólogos, adivinos y filósofos en la Atenas clásica, p. 189-195

María José HIDAlgo VEGA, La novela griega como vebiculo de propaganda religiosa, $\mathrm{p}$. 197-214

Pilar PAVON, Semejanzas iconográficas entre el culto al soberano belenístico y el culto imperial: apuntes para un estudio, p. 215-218

Fernando GASCO, Elio Aristides, Teodoro, p. 239-244.

Ugo BIANCHI (éd.), The Notion of "Religion " in Comparative Research. Selected Proceedings of the XVIth Congress of the International Association for the History of Religions, Rome, 3rd-8th September 1990, Roma, 'L'Erma' di Bretschneider, 1994.

Douglas R. EDWARDS, Religion, Politics, and Entertainment in the Graeco-Roman World: Chariton and His City Aphrodisias, p. 283-288

William E. MIERSE, The Interplay between Religlon, Politics, and Entertainment in the Graeco-Roman World: Archaeological and Literary Evidence, p. 289-294

Maria ROCCHI, Les monts coucou et la natssance d'Arès, p. 295-300

Renate SCHLESIER, Olympische Religion und Chtbontsche Religion, p. 301-310

Glovanni CASADIO, La nozione di religione nel de Iside et Osiride di Plutarco e lo studio scientifico della religione, p. 349-353

Silvia Maria CHIODI, Il concetto di religione nel de Iside et Osiride di Plutarco, p. 354-368

Luther H. MARTIN, Religion and Dream Theory in. Late Antiquity, p. 369-374

Frederick E. BRENK, Greek Epiphantes and Paul on the Road to Damaskos, p. 415-424

Cesare MAGAZZÙ, L'uso di religio nella polemica anti-pagana di Arnobio di Sicca, p. 441446

Concetta ALOE SPADA, L'uso di religio e religiones nella polemica antipagana di Lattanzio, p. $459-464$.

Fabio MORA, Per una tipologia del pollteismo, p. 823-828.

Pierre CaBanes (éd.), L'Illyrie méridionale et l'Épire dans l'antiquité. II. Actes du IIe colloque international de Clermont-Ferrand (25-27 octobve 1990), Paris, de Boccard, 1993, 243 p.

C. DE SIMONE, Il santuario di Dodona e la mantica greca piu antica: consideraztoni linguistico-culturali, p. 51-54

S. DAKARIS, A.Ph. CHRISTIDIS, J. VOKOTOPOUlou, Les lamelles oraculaires de Dodone et les villes de l'Éplre du Nord, p. 55-60

F. SALVIAT, Timodamos et son Gaulos. Oracles et marchands à Dodone, p. 61-64

Chr. TZOUVARA-SOULI, Common Cults in Epirus and Albania, p. 65-82 
Albio Cesare Cassio, Giovanni CeRRI (éds), L'inno tra rituale e letteratura nel mondo antico. Atti di un colloquio Napoli, 21-24 ottobre 1991, Roma, GEI, 1991, 312 p.

P. ANGELI BERNARDINI, L'inno agli del nella lirica corale greca e la sua destinazione sacrale, p. 85-94

A. GOSTOLI, L'inno nella citarodia greca arcaica, p. 95-105

S. GRANDOLINI, Canto processionale e culto nell'antica Grecia, p. 125-140

G.A. PRIVITERA, Aspetti musicall nella storla del ditirambo arcaico e tardo-arcatco, p. 141153

C.O. PAVESE, L'inno rapsodico. Analist tematica degli Inni omerici, p. 155-178

P. POCCETTI, Forma e tradizioni dell'inno magico nel mondo classico, p. 179-204

R. VELARDI, Le origini dell'inno in prosa tra V e IV secolo a.C. Menandro Retore e Platone, p. $205-231$

M. PIZZOCARO, L'inno di Epidauro alla Madre degli Dei, p. 233-251

R. PRETAGOSTINI, Rito e letteratura negli inni 'drammatici' di Callimaco, p. 253-263.

Manuela GaRcia VAldes (éd.), Estudios sobre Plutarco: ideas religiosas. Actas del III Simposio Internacional sobre Plutarco. Oviedo, 30 de abril a 2 de mayo de 1992, Madrid, Ediciones Clásicas, 1994, XII+696 p.

Frederick E. BRENK, The Origin and the Return of the Soul in Plutarch, p. 3-24

Rosa María AGUILAR FERNANDEZ, El concepto de anathymiasis en Plutarco, p. 25-31

Jan OPSOMER, L'ame du monde et l'ame de l'bomme chez Plutarque, p. 33-49

Engracia DOMINGO GARCIA, El Pneuma como inspiractón, p. 51-56

Adelmo BARIGAZZI, L'arresto della freccia del tempo (De facie in orbe lunae $941 \mathrm{Ass}$.), p. $57-69$

José GARCIA LOPEZ, Fenomenología religiosa en la Vida de Licurgo, p. 71-80

Marcos MARTINEZ FERNANDEZ, Islas escatológicas en Plutarco, p. 81-108

Luis GARCIA MORENO, Plutarco, Delfos y la Mitología celta, p. 109-113

Sven Tage TEODORSSON, The Psychology of De facte and De virtute morali, p. 115-122

Santiago GONZALEZ ESCUDERO, Evisal $\mu$ ovla divina en el Colotes de Plutarco, p. 123-130

Emilio Angel GARCIA GARCIA, La idea de daimon en Plutarco, p. 131-135

Domingo PLACIDO SUAREZ, Teseo: la tradición y la renovactón en la religlosidad de Plutarco, p. 137-143

José Antonio FERNANDEz DElgado, Plutarco como fuente de los oráculos, p. 145-156

Manuel CEREZO MAGAN, La superstición segín Plutarco de Queronea ¿Otra forma de religiōn?, p. 157-168

Francisco Javier GOMEZ ESPELOSIN, Los elementos religiosos de la Vita Alexandri, p. 169-178 José Joaquín CAEROLS PEREZ, Sibilas y oráculos sibilinos en Plutarco, p.179-188

Carmen BARRIGON FUENTES, Angel RUIZ PEREZ, Observaciones sobre algunos oráculos de Plutarco ( $P W$ 41 y 52), p. 189-197

Ascensión VERA MUÑOZ, La $\mu \epsilon \tau a \beta 0 \lambda \eta x$ del delincuente en Ser num. vind., p. 199-203

Luigi TORRACA, Il problema del male nella teologta plutarchea, p. 205-222

Gonzalo DEL. CERRO CALDERON, El problema del mal y de la providencla en Plutarco y en la Biblia, p. 223-234

Serafín BODELON GARCIA, Elementos religiosos en la Vida de Cicerón según Plutarco, p. $235-246$

Miguel BRIONEZ ARTACHO, El elemento religioso en la Vida de Arístides de Plutarco, p. $247-251$

Fernando LABAJOS BRIONES, Antonio LOZANO Y DE CASTRO, La religión en Plutarco: Theoria - estética a la escucba de la divinidad, p. 253-260 
María Teresa LOZANO DE CASTRO, La obra de Plutarco de Queronea fundamento de la verdad religiosa y espiritual, p. 261-264

Luciano PEREZ VILATELA, Artemís y Dioniso Ibéricos: Apelación a Plutarco, p. 265-272

Luc VAN DER STOCKT, Plutarch on the Poetry of Prophecy, p. 321-329

Vicente DOMINGUEZ GARCIA, ¿Plutarco responsable del ateísmo de Evémero de Mesene? Comentarlo $a$ De Iside et Osiride $360 A-B$, p. 509-514

Ana Isabel OSORIO VIDAURRE, Dialéctica razón - religión en el pensamiento de Plutarco, p. 551-559

Félix FORTEA LOPEZ, Némesis en la obra de Plutarco de Queronea, p. 561-573

Manuela GARCIA VALDEZ, Píndaro en Plutarco: los dioses, p. 585-604

María Concepción MORALES OTAL, El mito en la Vida de Teseo, p. 625-630

Angeles DURAN LOPEZ, El mito de Gorglas en la Consolatio ad Apollonium, p. 643-650.

Albert Machin, Lucien Pernée, Sopbocle; le texte, les personnages. Actes du colloque international d'Aix-en-Provence 10, 11 et 12 janvier 1992, Aix-en-Provence, Publications de l'Université de Provence, 1993, 388 p.

J.M. LUCAS, Le mythe de Danaé et de Persée chez Sophocle, p. 35-48

D. PRALON, L'éloge d'Aphrodtte (Sophocle, 941 Radi), p. 125-131

A. MOREAU, Les prophéties de Tirésias : un devin trop bumain (GEd. Roi, 300-462), p. 219232

Ch. SEGAL, Les oracles des Trachiniennes et les rites renversés du marlage, p. 233-241

\section{Agostino Masaracchia (éd.), Orfeo e l'orfismo. Atti del Seminario Nazionale (Roma-Perugia 1985-1991), Roma, Gruppo Editoriale Internazionale, 1993, 652 p., 22 fig.}

Agostino MASARACCHIA, Introduzione, p. 13-26

Gabriella RICCIARDELLI APICELLA, Le teogone orfiche nell'ambito delle teogonte greche, p. $27-51$

Alessandro PARDINI, L Ornitogonia (Ar. Av. 693 sgg.) tra serio e faceto: premessa letteraria al suo studio storico-religioso, p. 53-65

Sabrina COLABELLA, Sul paptro di Derveni, p. 67-75

Gabriella IACOBACCI, Orfeo argonauta. Apollonio Rodio 1, 494-511, p. 77-92

Ippolita AVANZINI, Il fr. $86 \mathrm{~K}$.: una nuova ipotesi, p. 93-99

Massimo DI MARCO, Dioniso ed Orfeo nelle Bassaridi di Escbilo, p. 101-153

Adele-Teresa CozzolI, Euripide, Cretesi, fr. $472 \mathrm{~N}^{2}$ (79 Austin), p. 155-172

Agostino MASARACCHIA, Orfeo e gli 'orfict' in Platone, p. 173-197

Angela DI FABIO, La catabasi di Orfeo in Ermesianatte di Colofone: metamorfost di un mito, p. 199-210

Adriano PENNACINI, La narrazione patetica di Virgilio: Orfeo nell'Ade, p. 211-218

Carlo SANTINI, Orfeo come personaggto delle Metamorfosi e la sua storia racontata da Ovidio, p. 219-233

Giuseppe GIANGRANDE, La lamina orfica di Hipponion, p. 235-248

Gabriella IACOBACCI, La laminetia aurea di Hipponion: osservazioni dialettologiche, p. 249264

Raffaele LUISELLI, Contributo all'interpretazione delle Argonautiche orfiche: Studlo sul proemio, p. $265-307$

Nino Borsellino, La voce e lo sguardo. Orfeo nella Fabula del Poliziano, p. 309-317

Mario MARTELLI, Il mito d'Orfeo nell'età laurenziana, p. 319-351

Gennaro SAVARESE, Orfismo a Roma tra filologia e cabala (1505-1532), p. 353-366

Luigi M. LOMBARDI SATRIANI - Domenico SCAFOGLIO, Orfeo e Ptulcinella, p. 367-382

Franca ANGELINI, Orfeo allo speccbto, p. 383-398 
Antonia F. G. ALESSIO CAVARRETTA, Diffusione diacronica dell'iconografia di Orfeo in ambiente occldentale, p. 399-407

Crescenzo FIORE, Aspetti sciamanici di Orfeo, p. 409-424

Fiorella GIACALONE, Tracce orfiche nella letteratura popolare meridionale, p. 425-445

Guy LANOUE, Orpheus in the netberworld in the plateau of western north America: the voyage of Pent, p. 447-485

Maricla BogGIO, Lo sguardo di Orfeo, p. 489-623.

\section{Ouvrages collectifs et mélanges}

Siegmar Döpp (éd.), Karnevaleske Pbänomene in antiken und nachantiken Kulturen und Literaturen. Stätten und Formen der Kommunikation im Altertum I, Trier, Wissenschaftlicher Verlag Trier, 1993, 320 p., 21 fig.

W. BURKERT, Kronia-Feste und ibr altorientalischer Hintergrund, p. 11-30

W. RÖSLER, Über Aischrologte tm archaischen und klassiscben Griechenland, p. 75-97

B. TEUBER, Zur Scbretbkunst eines Zirkusreiters: Karnevaleskes Erzablen im 'Goldenen Esel' des Apuleius und die Sorge um sich in der antiken Ethik, p. 179-238

W. MEZger, Antike Motive und Elemente in der Fastnacht des Spatmittelalters? Zu Kontinuitat und Diskontinuitat der Traditionen des klassischen Altertums, p. 239-264.

\section{Carol Dougherty, Leslie Kurke (éds), Cultural Poetics in Archaic} Greece. Cult, Performance, Politics, Cambridge, Cambridge Univ. Press, 1993, XVI+266 p., 30 fig.

I.. MORRIS, Poetics of Power: The Interpretation of Ritual Action in Arcbaic Greece, p. 15-45

C. ANTONACCIO, The Archaeology of Ancestors, p. 46-70

R.H. SINOS, Divine Selection: Eplphany and Polittcs in Archaic Greece, p. 73-91

H.A. SHAPIRO, Hipparchos and the Rhapsodes, p. 92-107

D. BOEDEKER, Hero Cult and Politics in Herodotus: The Bones of Orestes, p. 164-177

Attilio MAstrocinque (éd.), I grandi santuari della Grecia e l'occidente, Trento, Dipartimento di scienze filologiche e storiche, 1993, 158 p., nombr. ill. (Labirinti, 3).

A. ALONI, Introduzione, L'invenztone di Delo, p. 13-30

F. COARELLI, I Tarquint e Delfi, p. 31-42

G. COLONNA, Doni di Etruscbi e di altri barbart occidentali nei santuari panellentct, p. 4367

J. DE LA GENIÈRE, L'intzio della romanizzazione a Claros, p. 69-79

M. DE VOS, Il templo di Iside in via Labicana a Roma, p. 81-91

M. GIANGIULIO, Le città di Magna Grecia o Olimpia in età arcatca, p. 93-118

A. MASTROCINQUE, Artistl samil in occidente, p. 119-135

U. SINN, L'attività dell'imperatore Nerone ad Olimpia. Risultati e prospettive del nuovi scaul, p. $137-147$

M. SORDI, I rapporti fra Roma e Delfi e la dectma, p. 149-158. 